\title{
Vers une gouvernance apprenante
}

Towards a participatory governance

Hacia una gobernanza de aprendizaje

Anne-Marie Bardi et Jean-Pierre Véran

\section{(2) OpenEdition}

\section{Journals}

Édition électronique

URL : https://journals.openedition.org/ries/9357

DOI : 10.4000/ries.9357

ISSN : 2261-4265

\section{Éditeur}

France Education international

Édition imprimée

Date de publication : 30 avril 2020

Pagination : 105-112

ISBN : 978-2-85420-626-5

ISSN : 1254-4590

\section{Référence électronique}

Anne-Marie Bardi et Jean-Pierre Véran, « Vers une gouvernance apprenante », Revue internationale d'éducation de Sèvres [En ligne], 83 | avril 2020, mis en ligne le 17 juin 2020, consulté le 25 juin 2021. URL : http://journals.openedition.org/ries/9357 ; DOI : https://doi.org/10.4000/ries.9357 


\title{
Vers une gouvernance apprenante
}

\author{
Anne-Marie Bardi \\ Inspectrice générale $(H)$ \\ Jean-Pierre Véran \\ Inspecteur d'académie \\ Inspecteur pédagogique régional $(H)$
}

Le choix des mots a son importance, et leur histoire également. Si le gouvernement consiste depuis l'antiquité, comme l'indique le dictionnaire historique de la langue française Robert, à " diriger quelque chose ou quelqu'un ", initialement un navire, la gouvernance est réapparue depuis la fin du $\mathrm{Xx}^{\mathrm{e}}$ siècle pour désigner la complexité d'un régime de pilotage caractérisé en 1995 par Willy Brandt au G20 comme « un processus continu de coopération et d'accommodement entre des intérêts divers et conflictuels ». Les termes de "gouvernance éducative » ou " gouvernance scolaire » se sont imposés au début du Xxi siècle, à différentes échelles, depuis les évaluations internationales des acquis des élèves jusqu'au niveau de l'unité d'enseignement.

\section{LE GOUVERNEMENT ÉDUCATIF FACE AUX QUESTIONS PRATIQUES}

La première nécessité d' « articuler les gouvernances » pourrait venir de ces questions d'échelles. Quel lien entre les résultats des enquêtes internationales, dont PISA constitue une figure emblématique, et les choix pédagogiques établis à l'échelle d'une classe ou d'une école ? Mais cette articulation entre le global et le local n'est qu'un des facteurs de complexité d'une typologie des gouvernances. Comment articuler à l'échelle d'une unité d'enseignement les intérêts divers et éventuellement conflictuels des élèves, des enseignants, des parents ? Comment articuler, à l'échelle d'un territoire, ce qui relève d'orientations de politique éducative prises à l'échelon confédéral ou cantonal, comme en Suisse, fédéral, étatique et local, comme aux États-Unis d'Amérique ? La compétence éducative est-elle partagée entre plusieurs décideurs ou confiée à l'un d'entre eux ? La réponse à cette question n'est jamais univoque. D’où cette difficulté signalée par Bill et Melinda Gates (2018) :

Pour obtenir un changement significatif (dans les apprentissages des élèves), il faut parvenir à un consensus entre toute une série de décideurs : les autorités publiques, les commissions scolaires, les administrateurs, les enseignants et les parents d'élèves.

Mais ceci n'est encore qu'une première approche. L'articulation des gouvernances n'est pas seulement une question d'échelle, elle est aussi une affaire de nature. Selon quels critères pilote-t-on, seul ou à plusieurs, l'éducation, à quelque échelle que ce soit ? Choisit-on de privilégier l'excellence du rapport investissement/résultat, en s'imposant prioritairement, voire exclusivement, d'optimiser la dépense d'éducation? On sera alors dans une gouvernance instrumentale, qui n'anticipe pas toujours l'impact 
de ses décisions, comme l'a montré la chercheuse Lisbeth Lundahl (2019) ${ }^{1}$, à propos du « chèque scolarité » instauré en Suède. Choisit-on de privilégier les espaces de concertation, de consultation avant la prise de décision ? On sera là dans une gouvernance procédurale. Choisit-on de référer chaque décision aux valeurs éducatives (équité, égalité des filles et des garçons, alliance éducative avec les parents...) ? On privilégiera alors une gouvernance morale, qui devra arbitrer souvent entre la recherche de l'excellence et celle du bien-être des élèves, comme Chae-chun Gim (2019) l'a montré en évoquant la situation en Corée du Sud. On le voit bien, il s'agit d'articuler au mieux chacune de ces gouvernances, car il faut tout à la fois optimiser les dépenses, garantir le caractère démocratique de la délibération et promouvoir les valeurs éducatives.

Et nous pourrions filer la métaphore navale : une bonne navigation nécessite de fixer un cap - quitte à savoir le modifier en fonction de l'environnement - et de tenir ce cap à l'aide du gouvernail. Mais il faut aussi savoir utiliser les énergies disponibles, le vent, les risées, les courants et faire en permanence un bon choix des jeux de voiles. Bien choisies, bien réglées, elles porteront vers la destination ; inadaptées, elles demeureront inefficaces... si elles ne font pas chavirer le navire.

Il en va de même pour le pilotage d'un système éducatif. Les forces en présence sont à mobiliser en ayant l'intelligence des effets produits et la visée de leur cohérence : à quoi affecter prioritairement les moyens financiers ? Sur quels axes développer la formation des acteurs ? Comment la gestion des affectations et des carrières peut-elle renforcer une impulsion ? Peut-on jouer sur la forme et sur le contenu des examens et des certifications ? Quelle place donner au contrôle, à l'évaluation, à l'auto-évaluation ? Choisit-on une éducation uniforme pour tous ou tient-on compte des minorités ethniques et culturelles, des enfants à besoins spécifiques, l'école pour tous devant être aussi l'école de chacun ? Si les réponses à ces questions sont données indépendamment les unes des autres, par des acteurs ignorants les uns des autres, alors il ne faudra pas s'étonner que le navire n'avance pas ! Cela se constate encore bien souvent...

\section{TENTER UN GOUVERNEMENT ÉDUCATIF PARTAGÉ}

Il est ainsi permis de douter que l'on soit passé de l'ère du gouvernement à celle de la gouvernance. Ne serait-ce que parce qu'on parle de gouvernances au pluriel et non de la gouvernance. Si l'on observe la manière dont se passent les choses à l'échelle d'un territoire supranational ou infranational, on peut facilement observer que l'on est passé non pas du gouvernement à la gouvernance, mais d'un gouvernement unique à un gouvernement partagé. Au sein de l'Union européenne, par exemple, on distingue des « compétences exclusives » de l'Union et des « compétences partagées » avec les États, répartissant ainsi le gouvernement entre différents échelons, selon le principe de subsidiarité, comme si toute question pouvait être

1. Cet article fait suite au colloque international « Conditions de réussite des réformes en éducation ", organisé par la Revue internationale d'éducation de Sèvres, 12-14 juin 2019, CIEP, et notamment à l'atelier 6 intitulé "Efficacité et gouvernance : des articulations complexes ? ", coordonné par les auteurs du présent article, où cinq études de cas ont été présentées par L. Lundahl (Suède), M. Jakubowski (Pologne), J.-R. Cytermann (France), C. Gim (Corée du Sud), M. Behrens (Suisse). Voir : [https://journals.openedition.org/ries/6658] 
résolue à un échelon territorial unique. Or la réalité, qu'elle soit politique ou éducative, ne se découpe pas selon des échelons territoriaux, car ces différents échelons sont interdépendants pour résoudre le problème posé. On voit bien comment, dans les pays de tradition confédérale, on éprouve aujourd'hui le besoin d'établir une concertation éducative pour déterminer des objectifs éducatifs communs à une échelle confédérale, même lorsque n'existe pas d'autorité en matière éducative à cette échelle. M. Behrens (2019), ancien directeur de l'Institut de recherche et de documentation pédagogique (IRDP) de Neuchâtel (Suisse), a finement analysé le caractère complexe de ce partage et les réticences qu'il suscite dans les cantons suisses. À l'inverse, dans des pays de tradition étatique centralisée, l'État applique une politique de décentralisation qui remet une partie des compétences éducatives à tel ou tel échelon infra-étatique, sans pour autant les remettre toutes, ce qui pourrait conduire par exemple à ce que théoriquement, en France, l'autorité départementale construise un collège sans que l'État n'y affecte d'enseignants.

L'ère du gouvernement partagé est effective, avec, derrière le jeu institutionnel apparent, le gouvernement bien réel des techniciens qui, à chaque échelon, informent le politique et orientent très fortement sa prise de décision. En matière d'éducation comme de manière plus générale en politique, on peut observer souvent que la technostructure prend le pas sur les décideurs politiques, privilégiant, comme l'a indiqué pour la France J.-R. Cytermann (2019), inspecteur général de l'administration, de l'éducation et de la recherche $(\mathrm{H})$, « un pilotage par la norme, avec une production massive de textes réglementaires et de circulaires ». C'est le règne des experts, qui concourt à renforcer la crise démocratique et la défiance à l'égard des gouvernements.

\section{L'ILLUSION DE LA « BONNE GOUVERNANCE »}

Comment passer d'un modèle de gouvernement partagé entre institutions, entre échelons de décisions, mais aussi entre décideurs et technostructure, à une véritable gouvernance?

Telle est l'une des questions posées par les débats conduits au cours du colloque "Conditions de réussite des réformes en éducation ", que prolonge ce numéro 83 de la Revue internationale d'éducation de Sèvres 2 .

Le colloque a permis d'abord de mettre en lumière des effets potentiellement pervers de ce que le nouveau management public puis des institutions internationales (Conseil de l'Europe, 2008) ont présenté comme des principes de «bonne gouvernance $»^{3}$. Il en va ainsi du libre choix de l'école par les parents, censé produire par l'émulation plus de qualité dans l'enseignement : on a mesuré la contre-productivité du « chèque scolarité » en Suède. Des comparaisons internationales menées par des chercheurs depuis le début des années 2010 ont en effet mis en lumière des effets négatifs de la mise en œuvre imprudente de certains principes du nouveau manage-

\footnotetext{
2. Voir note 2.

3. Citons notamment, parmi ces instruments à potentiel double tranchant, la reddition de comptes (accountability), l'efficacité (school effectivness), ce qui marche (what works). Efficacité et efficience (principe 3), obligation de rendre des comptes (principe 12) figurent ainsi parmi les douze principes de bonne gouvernance démocratique au niveau local, approuvés par une décision des ministres du Conseil de l'Europe (2008).
} 
ment public. Ainsi Romuald Normand (2011), comparant États-Unis, Angleterre et France, a-t-il pu montrer comment l'individualisation des carrières et la rémunération à la performance des enseignants peuvent nuire à leur travail d'équipe, comment la multiplication des tests standardisés pour mesurer les acquis des élèves peut réduire l'expérimentation pédagogique et nuire à la qualité des apprentissages, comment, pour être mieux classés en terme de résultats, des établissements scolaires peuvent se défaire des élèves "non rentables ", renforçant ainsi les inégalités sociales et raciales de réussite scolaire. Christian Maroy (2013), pour sa part, rassemblant des études conduites en Belgique, en France, en Hongrie, en Israël, au Québec et en Suisse, a mis en lumière le paradoxe de la recherche de la performance, qui se traduit par un contrôle administratif renforcé et qui substitue à la bureaucratie traditionnelle une hyper-bureaucratie qui, dans le travail des chefs d'établissement, fait prendre le pas à l'administratif sur le pédagogique. Ces phénomènes soulignent la nécessité absolue d'articuler les gouvernances, et de ne jamais privilégier exclusivement une gouvernance instrumentale ou procédurale en ne tenant pas suffisamment compte d'un des douze principes de bonne gouvernance démocratique retenus par le Conseil de l'Europe (2008) : le comportement éthique (principe 6), et, plus généralement, de recourir à l'arbitrage de la gouvernance morale fondée sur des valeurs éducatives.

Les débats du colloque ont aussi conduit à dépasser la définition de la gouvernance proposée par Willy Brandt. Si, en effet, on est en permanence à la recherche d'accommodements entre intérêts divers et conflictuels, on risque de se condamner à l'impuissance, tant ce processus dessine une porte étroite pour la définition et la conduite de la réforme. Comment trouver, entre des intérêts potentiellement conflictuels (ceux des parents, des élèves, des personnels, des entreprises, des collectivités, du territoire concerné...), un accommodement qui ne soit pas le maintien habile, sous les atours du changement, du statu quo ante, ce que le prince Salina, dans Le Guépard de Lampedusa, résume d'une formule célèbre : "Si nous voulons que tout reste tel que c'est, il faut que tout change ». N'y aurait-il pas là une clé d'explication à la "réformite » dont seraient atteints parfois les ministres de l'éducation : donner l'illusion du changement sans remettre en question les équilibres subtils entre pouvoir central et autorités locales, bureaucraties ministérielle et territoriales, bureaucraties syndicales, lobbies disciplinaires, groupes de pression des parents, experts du système scolaire, forces politiques et sociales mobilisant de puissantes manifestations ? Dans ce cadre, pour que l'«accommodement " soit possible, la réforme ne doit-elle pas être nécessairement limitée dans ses ambitions initiales et, par conséquent, encore plus limitée dans ses effets?

\section{Vers UNe GOUVERNANCE APPRENANTE}

Mais si, rompant avec les accommodements continus dans l'entre-soi des gouvernants et de leurs partenaires de jeu institutionnel, la gouvernance, au lieu d'être une navigation prudente, soucieuse d'éviter les écueils, consistait à rechercher les points d'appui et à en partager la connaissance entre les différents acteurs de l'éducation? S'il ne s'agissait plus de circonvenir ou de neutraliser les acteurs, les partenaires, en leur «vendant » une réforme, mais d'en faire les acteurs conscients, 
actifs du changement ? Si la gouvernance, c'était l'intelligence partagée ? L'enjeu n'est plus du tout alors dans la signature de la décision, mais dans le processus qui conduit à cette prise de décision.

Il serait temps d'harmoniser les finalités de l'école et son pilotage. Il s'agit à l'école de développer l'intelligence individuelle et collective des élèves ; il s'agit, dans sa gouvernance, de développer l'intelligence individuelle et collective de tous les acteurs. Penser la résolution des problèmes par le travail de groupes associant l'ensemble des acteurs, mettre au cœur de la réforme l'expérimentation, les projets pilotes, plutôt que d'imposer des organisations clés en mains décidées par une technostructure, prendre le temps d'évaluer, avant de généraliser, de tirer profit des échecs comme des réussites, plutôt que d'effacer une réforme par une autre ${ }^{4}$, faire du développement professionnel des personnels, des membres des associations, des réseaux contribuant à l'éducation et de l'empowerment ${ }^{5}$ des élèves des priorités et non des préoccupations subalternes, voici quelques pistes qui pourraient, en s'articulant, donner une véritable consistance à la gouvernance éducative.

En effet, n'est-il pas paradoxal que l'école ne soit pas encore partout une organisation apprenante, alors qu'elle aurait dû être la première d'entre elles ? N'est-ce pas un gaspillage colossal que dans certains pays, les enseignants puissent " jouer seulement de l'espace clos de la classe pour s'inventer des marges de manœuvre ", comme l'ont écrit en 2017 les auteurs d'un rapport destiné à la ministre de l'éducation nationale de leur pays (Bizot, Houzel, Taddei, 2017) ?

Quel contraste entre des pays où les établissements scolaires sont conçus pour laisser des espaces et du temps à la coopération, à la concertation entre les différents acteurs de l'école, où les élèves sont considérés comme acteurs à part entière et pas seulement comme des esprits à former, et d'autres où la forme scolaire condamne les enseignants et les élèves à l'isolement ! Denis Meuret (2009) a forgé à ce sujet le concept de modèle politique d'éducation : selon le modèle qui les inspire, certains pays ont un contexte éducatif plus ou moins favorable à la coopération des élèves et des acteurs de l'école.

Si réussir la conduite du changement suppose un établissement scolaire apprenant, on peut penser qu'une gouvernance apprenante de l'éducation rendrait cette conduite encore plus sûre. L'exemple donné par Chae-chun Gim de la réussite, en Corée du Sud, de la réforme dite du "semestre de liberté », introduite en 2013, est particulièrement éclairant. La meilleure preuve de sa réussite est que ce semestre est devenu en 2017 « année de liberté».

Les conditions de la réussite résident dans le fait que cette mesure n'a pas été imposée à tous d'en haut :

Le gouvernement central s'est contenté de présenter sa vision et l'orientation politique, encourageant les bureaux chargés de l'éducation et les établissements scolaires à prendre l'initiative en matière de décisions sur les contenus et méthodes spécifiques. (Gim, 2019)

4. Cytermann (2019) a ainsi notamment observé qu'en France «la mise en place de dispositifs pédagogiques s'accompagne rarement d'une évaluation au départ. Lorsque ces derniers font l'objet d'expérimentations, la généralisation se fait avant toute évaluation sérieuse des résultats. Et, très souvent, avant qu'on ait pu en mesurer les effets, un nouveau dispositif remplace le précèdent ou coexiste avec lui ».

5. Empowerment désigne le processus d'autonomisation et de responsabilisation dans l'apprentissage ou dans le développement professionnel. 
La première année, seuls une quarantaine d'établissements pilotes ont mis en œuvre le dispositif, qui s'est étendu d'année en année sur la base du volontariat, et a été progressivement généralisé, avec l'accompagnement des instituts de recherches en éducation. Quatre ans plus tard, on est passé ainsi à "l'année de liberté ». En quoi consiste cette mesure ? À laisser aux élèves un semestre, puis une année entière de respiration, pour qu'ils accomplissent les travaux, recherches, initiatives de leur choix, accompagnés par les enseignants, échappant ainsi aux rigueurs de la compétition et des examens, gage de l'excellence du système éducatif coréen.

Dans la mise en expérimentation, la généralisation progressive puis l'extension de ce programme à l'échelle d'une nation, on distingue nettement les ingrédients de ce que pourrait être une gouvernance apprenante. Elle commence par la vision politique (nécessité de se préoccuper aussi du bien-être des élèves), partagée entre gouvernement central et échelons locaux, repose sur l'empowerment des élèves, qui prennent la main sur leur semestre puis leur année scolaire de liberté. Elle passe par le libre engagement dans ce programme des enseignants constitués en équipes d'établissements, par l'appui des instituts nationaux de recherche, impliqués dans le suivi et la réussite du dispositif en formant aussi enseignants et parents, par le soutien des médias et de l'opinion publique et par des subventions gouvernementales encourageant la mise en œuvre du dispositif. On trouve ainsi articulées bien des figures de gouvernance évoquées au début de cet article. On est à la fois dans l'instrumental (les subventions), le procédural (le libre engagement des établissements), l'éthique (le bien-être des élèves), les ressources humaines et le développement professionnel (la formation des enseignants et des parents), l'évaluation (l'implication des instituts de recherche dans le suivi). Il ne s'agit pas d'une réforme cosmétique mais d'une réforme de fond, qui introduit dans une forme scolaire extrêmement rigide, scandée par l'évaluation permanente des élèves lors des examens académiques, un espace d'initiative et de liberté absolument novateur, qui transforme également en profondeur le rôle des enseignants...

On s'éloigne ici d'un gouvernement partagé de l'école, et on tend vers une gouvernance apprenante. Celle-ci ne serait-elle à la fois qu'une utopie et qu'une uchronie ou, bien au contraire, la seule manière de sortir des impasses du gouvernement partagé ? Lors du colloque, le chercheur et ancien vice-ministre de l'éducation M. Jakubowski a établi un lien entre la spectaculaire amélioration des performances des élèves polonais lors des évaluations internationales et les réformes des quinze premières années du siècle, fondées sur une gouvernance décentralisée, l'autonomie des établissements scolaires, le partage des responsabilités et l'implication des acteurs locaux. De même, le Coréen C. Gim, qui a occupé les mêmes fonctions, a souligné les effets positifs du semestre puis de l'année de liberté, non seulement sur le bien-être amélioré des élèves sud-coréens, mais aussi sur la qualité de leurs performances académiques.

Comme l'écrit Alain Bouvier (2017), la multiplication des instruments numériques d'information, de communication, de documentation, de travail partagé et de formation à distance et en ligne «favorise les coopérations, les co-constructions, les échanges d'information et de pratiques, le travail horizontal, aide à introduire de l'intelligence collective, multiplie les possibilités de mémorisation, de tris intelligents et de capitalisation d'acquis et de compétences ». Ces possibilités nouvelles amplifient la possibilité de passer du gouvernement partagé à une gouvernance apprenante. 
Un nouveau modèle de gouvernance émerge des établissements scolaires qui, face à des publics en difficulté scolaire ou mus par une volonté de faire progresser davantage leurs élèves (...) se caractérisent de la façon suivante : une conception aiguisée de l'éducabilité de tous les élèves partagée par tous les acteurs; la conviction de chaque acteur qu'une solution peut être trouvée en cas de difficulté non résolue, si l'on cherche ; la nécessité perçue de travailler en équipe pour se soutenir et chercher ensemble ; l'attribution d'un rôle éducatif à toutes les catégories de personnel composant l'établissement ; le rôle indispensable du chef d'établissement comme quelqu'un qui déploie toutes ses ressources pour faciliter le travail en équipe des enseignants et donner à tous les acteurs un rôle important et valorisé ; la volonté de montrer aux élèves et aux parents que l'établissement est éminemment professionnel, tant dans son personnel, que dans son équipement, son entretien et son cadre de vie. (Merhan, Jorro et De Ketele, 2015)

Il est donc permis d'envisager la gouvernance comme fondée non sur un gouvernement formellement partagé, dont on a largement perçu les limites, les contradictions et surtout les résultats décevants, mais sur quelques leviers bien identifiés.

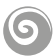

En régime de gouvernance apprenante, la réforme, le changement reposent sur des processus collectifs qui transforment les systèmes de pensée, les connaissances tacites en connaissances explicites partagées. Ils ne sauraient désormais se réduire à des textes réglementaires ni à des dispositifs institutionnels et juridiques pensés « en haut».

Les savoirs d'action font des acteurs les premiers experts, qui n'attendent plus les solutions d'une technostructure administrante qui bride leur initiative, au point qu'ils ne se sentent pas autorisés à innover, à explorer d'autres voies que les sentiers battus. Certes, il est plus risqué d'inventer sa pratique que d'appliquer des consignes, car on engage alors sa propre responsabilité : n'y aurait-il pas là une clé d'explication du maintien ici ou là d'un gouvernement hiérarchique de l'éducation fondé sur une chaîne de commandement?

En régime de gouvernance apprenante, les acteurs, conscients du fait que l'organisation influence les résultats, questionnent la forme scolaire, fût-elle la plus contraignante et la mieux installée dans les espaces, les temps et surtout les têtes. Toutes les routines qui emprisonnent et empêchent d'avancer ne sont plus autant d'impensés allant de soi, mais des formes historiquement datées et nécessairement dépassables.

En régime de gouvernance apprenante, c'en est fini des empilements de dispositifs sédimentés sans évaluation sincère de leur impact par les acteurs eux-mêmes. Un management pédagogique fondé sur le développement professionnel des acteurs privilégie le recrutement, l'accueil, l'accompagnement et la mobilité de ces derniers.

La gouvernance apprenante ainsi dessinée ne serait-elle pas, au Xxi ${ }^{\mathrm{e}}$ siècle, une condition nécessaire de la réussite des réformes en éducation? 


\section{BiblographiE}

BECCHETTI-BIZOT C., HOUZEL G. et TADDEÏ F. (2017). Vers une société apprenante, rapport sur la recherche et développement de l'éducation tout au long de la vie, avril. Paris : Ministère de l'éducation nationale, de l'enseignement supérieur et de la recherche. [https://bit.ly/2vRz7bL]

BEHRENS M. (2019). "En Suisse, quels leviers de gouvernance dans un système fondé sur la concordance? ». Revue internationale d'éducation de Sèvres [en ligne]. Colloque "Conditions de réussite des réformes en éducation », 12-14 juin 2019, CIEP. [http:// journals.openedition.org/ries/7701]

BOUVIER A. (2017). Pour le management pédagogique : un socle indispensable. Paris : Berger Levrault.

CONSEIL DE L'EUROPE (2018). 12 principes de bonne gouvernance démocratique, 2008. https://rm.coe.int/12-principes-de-bonne-gouvernance-democratique-et-outils-sur-labonne-/16808f0e23

CYTERMANN J.-R. (2019). " Comment réformer plus efficacement en France? » Revue internationale d'éducation de Sèvres [en ligne], colloque "Conditions de réussite des réformes en éducation », 12-14 juin 2019, CIEP. http://journals.openedition.org/ries/7714

GATES B. et GATES M. (2018). The ten toughest questions we get, Gates Notes, Our 2018 annual letter. https://www.gatesnotes.com/2018-Annual-Letter

GIM C. (2019). « Efficiency and governance: the case of South Korea ». Revue internationale d'éducation de Sèvres [en ligne]. Colloque 2019 "Conditions de réussite des réformes en éducation », 12-14 juin 2019, CIEP. [http://journals.openedition.org/ries/7769]

JAKUBOWSKI M. (2019). "Polish education reforms and governance structure ». Revue internationale d'éducation de Sèvres [en ligne]. Colloque 2019 "Conditions de réussite des réformes en éducation », 12-14 juin 2019, CIEP.[ http://journals.openedition.org/ ries/7801]

LUNDAHL L. (2019). "Swedish education reform: High ambitions and troubling results». Revue internationale d'éducation de Sèvres [en ligne]. Colloque 2019 "Conditions de réussite des réformes en éducation », 12-14 juin 2019, CIEP. [http://journals.openedition. org/ries/7843]

MAROY C., (dir.) (2014). L'école à l'épreuve de la performance, les politiques de régulation par les résultats. Louvain-la-Neuve : De Boeck.

MERHAND F., JORRO A., DE KETELE J.-M. (2015). Mutations éducatives et engagement professionnel. Louvain-la-Neuve : De Boeck supérieur.

MEURET D. (2009). Gouverner l'école. Une comparaison France/États-Unis, Paris : PUF, 2009.

NORMAND R. (2011). Gouverner la réussite scolaire, une arithmétique politique des inégalités. Peter Lang/ENS Lyon. 